Published in final edited form as:

ACS Appl Mater Interfaces. 2020 May 20; 12(20): 22499-22506. doi:10.1021/acsami.0c03001.

\title{
Dual-Color Plasmonic Nanosensor for Radiation Dosimetry
}

Yu Tao ${ }^{\nabla}$, Institute for Cancer Genetics, Columbia University, New York, New York 10032, United States; Laboratory of Biomaterials and Translational Medicine, The Third Affiliated Hospital of Sun Yatsen University, Guangzhou 510630, China

Mingqiang $\mathbf{L i}^{\nabla}$, Department of Biomedical Engineering, Columbia University, New York, New York 10027, United States; Laboratory of Biomaterials and Translational Medicine, The Third Affiliated Hospital of Sun Yat-sen University, Guangzhou 510630, China

Xiangyu Liu, Institute for Cancer Genetics, Columbia University, New York, New York 10032, United States

Kam W. Leong, Department of Biomedical Engineering, Columbia University, New York, New York 10027, United States

Jean Gautier ${ }^{\nabla}$, Institute for Cancer Genetics, Department of Genetics and Development, and Herbert Irving Comprehensive Cancer Center, Columbia University, New York, New York 10032, United States

\section{Shan Zha ${ }^{\nabla}$}

Institute for Cancer Genetics, Department of Pediatrics, Pathology and Cell Biology, Immunology and Microbiology, and Herbert Irving Comprehensive Cancer Center, Columbia University, New York, New York 10032, United States

\section{Abstract}

Radiation dosimeters are critical for accurately assessing the levels of radiation exposure of tumor sites and surrounding tissues and for optimizing therapeutic interventions as well as for monitoring environmental exposure. To fill the need for a simple, user-friendly, and inexpensive dosimeter, we designed an innovative colorimetric nanosensor-based assay for detecting ionizing radiation. We show that hydroxyl radicals generated by ionizing radiation can be used to etch gold nanorods (AuNRs) and silver nanoprisms (AgNPRs), yielding reproducible color changes for radiation dose detection in the range of 50-2000 rad, broad enough to cover doses used in hyperfractionated,

Corresponding Authors Phone: +1 212851 4564; jg130@cumc.columbia.edu; Fax: +1 212851 5284; Phone: +1 2128514779 ; sz2296@cumc.columbia.edu.

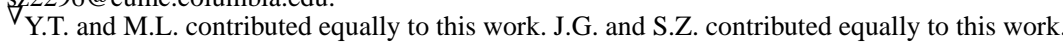
ASSOCIATED CONTENT Supporting Information

The Supporting Information is available free of charge at https://pubs.acs.org/doi/10.1021/acsami.0c03001.

Typical photographs and absorption spectra of AuNRs and AgNPRs after being stored for 6 months at room temperature, TEM image of AgNPRs following storage for 6 months at room temperature, absorption spectra of AuNRs and AgNPRs irradiated with X-ray after different time intervals (PDF)

The authors declare no competing financial interest. 
conventional, and hypofractionated radiotherapy. This range of doses detected by this assay correlates with radiation-induced DNA damage response in mammalian cells. Furthermore, this AuNR- and AgNPR-based sensing platform has been established in a paper format that can be readily adopted for a wide range of applications and translation.

\section{Graphical Abstract}

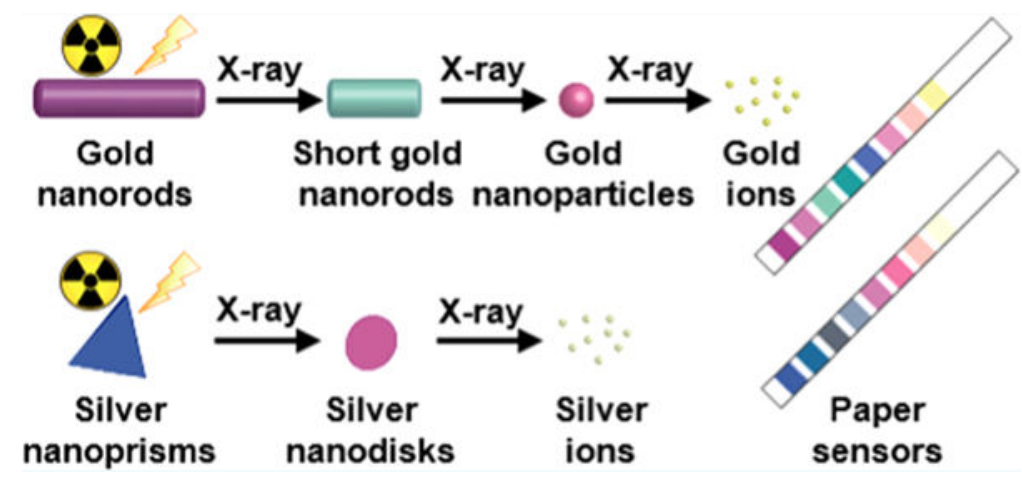

\section{Keywords}

radiation dosimetry; colorimetric detection; silver nanoprisms; gold nanorods; dual-color nanosensor

\section{INTRODUCTION}

Radiation therapy is a common and effective therapeutic modality for cancer treatment as more than half of all cancer patients receive radiotherapy as a part of their treatment.

Radiation therapy uses high-energy radiation (X-rays, $\gamma$-rays, electron beams, protons, and charged particles) to eliminate cancer cells, control their growth, and metastasis. ${ }^{1}$ However, if not properly controlled, radiation can also damage normal cells and induce rate-limiting toxicity in nearby healthy tissues. ${ }^{2}$ Radiation-induced skin pathologies, osteonecrosis, and other complications have been reported. In addition, cases of inaccurate dosing and accidental overdose owing to errors in machine calibration have also been known to occur. ${ }^{3}$ Therefore, to evaluate and minimize radiation-induced side effects, it is critical to develop radiation dosimeters to accurately measure radiation exposure both within the tumor and at surrounding tissues. In addition, radiation dosimetry is used to monitor environmental exposure and low-dose irradiation in the workplace or around industrial settings.

We sought to develop a simple, quick, and effective methodology for radiation dosimetry based on a nanoplasmic colorimetric assay. Similar sensing technologies have proven to have significant benefits over conventional sensors such as high biocompatibility, good stability, high sensitivity, and low cost. ${ }^{4,5}$ In contrast, traditional radiation dosimeters, such as the metal-oxide-semiconductor field-effect transistor (MOS-FET) dosimeter, thermoluminescent dosimeter (TLD), and quartz fiber dosimeter (QFD), ${ }^{6,7}$ usually require sophisticated and expensive fabrication technology, separate readout instruments, and professional operators. These drawbacks may limit their application in resource-constrained 
areas or countries. Additionally, since no equipment is needed to read the output signal except for the naked eye, it exhibits great potential for commercial portable daily life and medical applications.

Plasmonic nanoparticles, especially gold and silver nanoparticles, have been extensively applied to colorimetric bioanalytical assays for detecting various analytes. They exhibit strong localized surface plasmon resonance (LSPR) as well as characteristic size- and distance-dependent optical absorption properties. ${ }^{8,9}$ Rege and co-workers recently developed a gel-based colorimetric nanosensor dosimeter for proton radiation. ${ }^{10}$ In their assay, proton irradiation triggers the reduction of gold ions to gold nanoparticles within the gel matrix with a detection range of 0-3 Gy. Radiation-induced color changes of this platform range from colorless to red, which may be difficult to distinguish at low doses. Recently, gold nanoparticles of various shapes have been synthesized. ${ }^{11,12}$ Among anisotropic gold nanoparticles, gold nanorods (AuNRs) in particular have received a great deal of attention owing to their simple synthesis and modification process, uniquely tunable optical properties, and excellent thermal sensitivity. ${ }^{13,14}$ Multiple applications including medical imaging, cancer therapy, and bioanalysis have been proposed. ${ }^{15,16}$ AuNRs exhibit two optical absorption peaks arising from strong LSPR characteristics in the transverse and longitudinal directions. Specifically, the longitudinal LSPR can be tailored to absorb the light-spanning wavelength range from visible to near-infrared of up to $1600 \mathrm{~nm}$ by modulating the AuNR length to diameter ratio. ${ }^{17}$ The longitudinal LSPR peaks of AuNRs are extremely sensitive to minute changes in the aspect ratio of AuNRs, ${ }^{18}$ and the absorption peak shifts corresponding to the color changes can be precisely tuned by altering the aspect ratio by etching of the AuNRs. ${ }^{19}$ Silver-based nanoparticles can also be utilized as a colorimetric sensor owing to their morphology-dependent optical properties. ${ }^{20,21}$ In particular, triangular silver nanoprisms (AgNPRs) containing three sharp vertices exhibit unique resonance optical characteristics. ${ }^{22,23}$ Etching of the AgNPRs induces a blue shift of the LSPR peak concomitant with the shape changes of the nanoprisms into round nanodiscs. 24,25 Previous studies indicated that AuNRs and AgNPRs could be efficiently etched by reactive oxygen species. ${ }^{26}$ Because of these unique features, we decided to design AuNRand AgNPR-based wavelength-variation sensing platforms for effective radiation detection based on the radiation-induced etching of the nanoparticles. To the best of our knowledge, no work about AuNR- and AgNPR-based colorimetric radiation detection has been reported so far, making this system the first example of the usage of AuNRs and AgNPRs as an efficient radiation dosimeter.

\section{EXPERIMENTAL SECTION}

\subsection{Operative and Preferred Ranges of Process Parameters.}

The JL Shepherd Mark I irradiator was utilized to irradiate samples at a dose rate of 1.19 Gy/min. Then, the prepared silver nanoprisms were radiated at doses of 0 (control), 0.5, 1 , $1.5,2,2.5,3,4$, and $5 \mathrm{~Gy}$. The prepared gold nanorod samples were irradiated at doses of 0 (control), 1, 3, 5, 8, 10, 15, 20, 25, and $30 \mathrm{~Gy}$. After irradiation, the samples were kept in dark for $2 \mathrm{~h}$ before measured with a UV-vis spectrophotometer. 


\subsection{Preparation of the Gold Nanorod- and Silver Nanoprism-Based Paper Sensor.}

Adsorption of gold nanorods and silver nanoprisms on the paper substrate was achieved by immersing the filter paper in gold nanorod or silver nanoprism solution for $2 \mathrm{~h}$ and then airdried.

\subsection{Cell Cultures.}

Murine embryonic fibroblasts (MEFs) were cultured in Dulbecco's modified Eagle medium (DMEM) supplemented with heat-inactivated fetal bovine serum (FBS) and $200 \mathrm{unit} / \mathrm{mL}$ penicillin/streptomycin plus $1 \times$ nonessential amino acids (GIBCO). The cells were kept at $37{ }^{\circ} \mathrm{C}$ in a humidified atmosphere containing $5 \% \mathrm{CO}_{2}$.

\subsection{Western Blotting.}

Cell lysates were prepared in RIPA buffer (50 mM Tris pH 8.0, 1\% NP40 0.1\% SDS, 150 $\mathrm{mM} \mathrm{NaCl}, 0.5 \% \mathrm{Na}$ deoxycholate, and the fresh proteinase inhibitor cocktail). Protein extracts were analyzed by Western blotting according to standard procedures using primary antibodies specific for KAP1 (TIF1 $\beta$, C42G12 Cell Signaling, \#4124), phosphor-KAP1 (A300-767A, Bethyl Laboratories), and $a$-tubulin (CP06, Calbiochem). HRP-conjugated antimouse and -rabbit secondary antibodies were utilized and the signal was detected using an ECL Western blotting detection system.

\section{RESULTS AND DISCUSSION}

The working principle of AuNR and AgNPR etching-based colorimetric assay for radiation dosimetry is illustrated in Figure 1. Ionizing radiation induces water splitting to generate oxidizing hydroxyl radicals $(\mathrm{OH} \bullet)$, which efficiently shorten the length of AuNRs (Figure 1a) as well as etch triangular AgNPRs into smaller round Ag nanodisks (Figure 1b), thereby triggering substantial blue shifts of LSPR peaks and corresponding color changes of AuNRs (burgundy $\rightarrow$ blue $\rightarrow$ green $\rightarrow$ pink $\rightarrow$ pale yellow) and AgNPRs (blue $\rightarrow$ purple $\rightarrow$ red $\rightarrow$ colorless). ${ }^{24}$ The changes in the morphology are dose-dependent, leading to a gradation of color variation that allows detection of radiation doses by direct colorimetric visualization.

AuNRs were coated with cetyltrimethylammonium bromide (CTAB), which facilitates the generation of nanorods and protects AuNRs from aggregating in solution. CTAB has higher coverage density along the long-side facets than the tip facets of AuNRs, resulting in less passivation and higher accessibility to irradiation at the tips of AuNRs. ${ }^{27}$ Therefore, etching of AuNRs initiates preferentially at the more exposed tip facets, resulting in nanorod shortening (Figure 1a). Accordingly, the longitudinal LSPR peak, which is highly dependent on the aspect ratio change of AuNRs, progressively blue-shifted to shorter wavelengths, consequently leading to the color change from burgundy to bluish green. ${ }^{28}$ Further radiation yields spherical gold nanoparticles, which in solution range from pink to pale yellow. The progressive color changes and concomitant plasmonic shifts are the consequence of the progressive etching of AuNRs by following ionizing radiation. Conveniently, these color changes are apparent even to the naked eye. ${ }^{29}$ Similarly, radiation-mediated etching of AgNPRs leads to the formation of smaller round Ag nanodisks (Figure 1b), and the 
concomitant blue shift of the LSPR band results in significant color variations (blue $\rightarrow$ purple $\rightarrow$ red $\rightarrow$ colorless). These characteristics make the AuNRs and AgNPRs ideal for designing colorimetric-based sensors for radiation dosimetry.

AuNRs and AgNPRs were synthesized according to the published protocols with slight modification ${ }^{24,30}$ (see details in the Supporting Information section). We first assessed the properties of the nanoparticles. We monitored the UV-vis absorption spectra (Figure 2a,d), the $\zeta$-potentials (Figure $2 \mathrm{~b}, \mathrm{e}$ ), and the size and shape by transmission electron microscopy (TEM) (Figure 2c,f). The absorbance spectrum in Figure 2a shows the characteristic transverse and longitudinal bands of AuNRs at 520 and $760 \mathrm{~nm}$, respectively. $\zeta$-Potential measurements indicate that the AuNRs are highly positively charged $(42.3 \mathrm{mV})$ consistent with the formation of the CTAB bilayer around AuNRs (Figure 2b). ${ }^{30}$ The measurement of the hydrodynamic size of the AuNRs by dynamic light scattering (DLS) reveals the size of $55 \pm 3.20 \mathrm{~nm}$ (data not shown). The morphology and size of AuNRs were further assessed by TEM. Figure 2c shows a representative TEM image of AuNRs, which are dispersed and show uniform size distribution with an aspect ratio of approximately 3.7. Similarly, Figure $2 \mathrm{~d}$ shows the UV-vis absorption spectrum of AgNPRs with two characteristic bands at 665 $\mathrm{nm}$ ascribed to in-plane dipole resonance and at $335 \mathrm{~nm}$ corresponding to out-of-plane quadrupolar resonance. ${ }^{31}$ We further characterized AgNPR morphology by combining DLS and TEM analyses. Size and $\zeta$-potential measurements (Figure 2e) indicate that the average hydrodynamic size and surface charge are $42 \mathrm{~nm}$ and $-15 \mathrm{mV}$, respectively. The representative TEM picture in Figure $2 \mathrm{f}$ shows that AgNPRs exhibited a triangular prism structure with an edge length between 25 and $45 \mathrm{~nm}$. Notably, AuNR and AgNPR nanoparticles were stable for at least 6 months at room temperature (Figures S1 and S2).

Next, we assessed the behavior of AuNRs and AgNPRs by following ionizing radiation exposure by monitoring the absorption spectra. We sought to assess how radiation-induced etching correlates with shifts in spectra. We observed a progressive shift in the absorbance with increasing radiation doses (Figure 3a), with gradual bleaching and shifting from 760 $\mathrm{nm}$ toward blue light due to the shortening of AuNRs as the etching process progresses. The wavelength shifts were time-dependent, reached a constant value after $2 \mathrm{~h}$ and did not further increase for at least $160 \mathrm{~h}$ (Figures 4 and S3). Therefore, $2 \mathrm{~h}$ incubation time was selected. The etching process is highly radiation dose dependent and the wavelength shift increases linearly between 1 and 20 Gy (Figure 3b). Higher radiation doses induce stronger bleaching and larger blue shifts of the LSPR peak, a prerequisite for developing an efficient radiation detector. In addition, radiation-induced LSPR peak shifts are highly reproducible within the 1-20 Gy range, where radiation doses could be quantitatively evaluated. Although we could detect absorbance changes for AuNRs in the 1-30 Gy range, the absorbance did not significantly shift below $1 \mathrm{~Gy}$, showing the lower limit of detection of the AuNR-based nanosensor. Next, we assessed the impact of ionizing radiation on AgNPRs. Figure 3c shows the absorbance spectra of AgNPRs at increasing radiation doses. Similar to what we observed for AuNRs, the maximum absorbance of AgNPRs gradually shifted to shorter wavelengths with a concomitant decrease in absorbance intensity at radiation doses ranging from 0.5 to $5 \mathrm{~Gy}$, establishing that AgNPR etching is also proportional to radiation doses. Notably, the increase in LSPR peak shift was linear within the $0.5-3$ Gy radiation range, where the radiation doses could be accurately measured, with a lower detection limit of 0.5 
Gy. Thus, the AgNPR further extends the detection limits of AuNRs to lower ranges important for biological applications. The higher detection sensitivity of AgNPRs compared to AuNRs can be attributed to the inherent susceptibility of triangular AgNPRs to reactive oxygen species (hydroxyl radical and $\mathrm{H}_{2} \mathrm{O}_{2}$ ) generated by ionizing radiation.

We originally hypothesized (see above) that progressive etching of the nanorods and/or nanoparticles and their subsequent size changes should take place upon irradiation. Next, we used DLS and TEM measurements to follow the changes in the shape and size of AuNRs and AgNPRs following irradiation. Figure 5a shows TEM images of AuNRs subjected to increasing radiation doses. Etching of AuNRs was proportional to radiation dosage. AuNRs were etched along the longitudinal direction, resulting in the shortening of AuNRs. Ultimately, AuNRs were transformed from nanorods into smaller spherical nanoparticles and even to complete dissolution at high radiation doses. This observation was consistent with the previous reports, confirming that the lower surface coverage of surfactant CTAB at the tips of AuNRs was responsible for preferential etching along the length of the nanorod. 32,33 Similarly, we could observe morphological transitions of AgNPRs during radiation by TEM (Figure 5d). The morphology of the AgNPRs gradually changed from triangle nanosheets to round nanodisks accompanied by size reduction following irradiation, ultimately resulting in very small silver nanoparticles at high radiation doses. This could explain the significant absorption spectral shifts and color changes of AgNPRs during the etching process. Using DLS and $\zeta$-potential measurements (Figure $5 \mathrm{c}$,f), we also found a reduction in the hydrodynamic size and $\zeta$-potential of the AuNRs and AgNPRs with increasing radiation doses. High radiation doses produced a larger number of free radicals as compared to lower radiation doses, which could lead to more severe etching and the formation of smaller metal nanoparticles. High radiation-mediated etching of AuNRs and AgNPRs finally led to the formation of metal ions, which was confirmed by X-ray photon spectroscopy (XPS) analysis (Figure 6).

Prompted by the sensitivity of the AuNR- and AgNPR-based biosensing platform in solution, we next attempted to develop a simple paper-based biosensor platform for onsite naked-eye detection of radiation doses using AuNRs and AgNPRs as the colorimetric reporters. Paper-based platforms are attractive and user-friendly methodologies in analytical and clinical chemistry applied to food quality testing, public health and environmental monitoring, "point-of-care" medical diagnosis, and forensic sciences. ${ }^{34-36}$ As analytical support, the paper provides great advantages including low cost, portability, compatibility, and ease of usage, storage, transport, and modification. ${ }^{37,38}$ Notably, the utilization of colorimetric AuNR- and AgNPR-based paper tests for the detection of the radiation dosage has not been assessed.

To adapt the assay to paper format, AuNRs and AgNPRs were immobilized by immersing the filter paper in AuNR and AgNPR solution for $2 \mathrm{~h}$ and then air-dried. ${ }^{39}$ For each of the metal nanoparticles, colorimetric paper tests were performed over the same ranges of radiation doses, as shown in Figure 7. We observed that the color of AuNRs gradually changed from burgundy to bluish green, then pink, and finally to pale yellow with increasing radiation doses (Figure 7a). Similarly, immobilized AgNPRs exhibited color-change properties in response to ionizing radiation. Figure $7 \mathrm{~b}$ indicated that the changes in color 
could be detected even with $0.5 \mathrm{~Gy}$ increments of the radiation dose. Importantly, the paper color did not change over time in the absence of radiation. Together, these results indicated that the paper test was suitable to determine radiation doses in the $0.5-20 \mathrm{~Gy}$ range. This range covered therapeutic doses employed in hyperfractionated ( $0.5-1.8 \mathrm{~Gy})$, conventional (1.8-2.2 Gy), and hypofractionated (3-10 Gy) radiotherapy., ${ }^{3,40}$ Importantly, paper-based assays using colorimetric nanoparticles circumvented the requirements for expensive equipment and provided low detection limits, offering a user-friendly alternative for convenient onsite assay and widespread implementation.

We next evaluated how the radiation doses detected by AuNR and AgNPR dosimetry correlated with the biological impact of radiation or oxidative damage detected on cells. In cells, ionizing radiation (IR) triggers the production of excess reactive oxygen species (ROS), including hydroxyl radicals, which subsequently result in DNA damage. IR-induced DNA double-stranded breaks (DSBs) activate the ataxia-telangiectasia mutated (ATM) protein kinase, which in turn phosphorylates numerous substrates. ${ }^{41}$ Among the ATM substrates, KAP1 (also called tripartite motif-containing 28, TRIM28) is phosphorylated at serine 824 by ATM ${ }^{42}$ facilitating DNA repair together with other DNA damage proteins. ${ }^{43}$ First, we assessed the levels of phosphorylated KAP1 at serine 824 (pKAP1) after varying doses of IR exposure as an indicator of DNA damage response (Figure 8a). As anticipated, we observed increasing pKAP1 signals by Western blotting with increasing IR doses, suggesting a concomitant increase of ATM activation. In independent experiments, cells were treated with increasing concentrations of organic peroxide tert-butyl hydroperoxide (TBH), which generates ROS in the absence of IR. This resulted in a similar increase in the pKAP1 signal (Figure 8b). This validates our approach to use AuNR- and AgNPR-based colorimetric nanosensors for radiation dosimetry within radiation ranges that cause measurable DNA damage in cells. By combining AuNR- and AgNPR-based colorimetric dosimeters, our method is capable of quantifying a broad range of environmental damage, including but not limited to, radiation- and ROS-inducing agents. This, in turn, may open up new research avenues for the characterization of DNA damage and ROS.

\section{CONCLUSIONS}

In summary, we have established a simple yet powerful nanosensor for detecting ionizing radiation through etching of AuNRs and AgNPRs induced by hydroxyl radicals generated by ionizing radiation, yielding reproducible color changes. This colorimetric sensor can detect radiation doses ranging from 0.5 to $20 \mathrm{~Gy}$. This approach allows for visible detection of radiation doses with high sensitivity and excellent reproducibility. In addition, the unique properties of AuNRs and AgNPRs, such as facile synthesis and modification, low cost, uniquely tunable optical properties, and excellent thermal sensitivity and aqueous solubility, add benefits to the sensing platform. Moreover, this AuNR- and AgNPR-based sensing platform has been established as a paper test. The translational potential of this dosimeter includes assisting oncologists with treatment planning and improving the therapy effectiveness, thus resulting in better patient outcomes. Taken together, this AuNR- and AgNPR-based colorimetric sensing platform presents a new dosimetry of therapeutic radiation dose with potential applications in radiation therapy. 


\section{Supplementary Material}

Refer to Web version on PubMed Central for supplementary material.

\section{Acknowledgments}

We thank the members of the Gautier, Leong, and Zha lab for technical assistance and helpful discussions. This work is in part supported by the National Institute of Health grants CA174653 to J.G. and S.Z., R01CA158073 to S.Z., and CA197606 to J.G. S.Z. is a recipient of the Leukemia Lymphoma Society Scholar Award. Additional experiments added during the revision were conducted in the Third Affiliated Hospital of Sun Yat-sen University and financially supported by the National Natural Science Foundation of China (21907113, 51903256) and the Guangdong Province Science and Technology Innovation Special Fund (International Scientific Cooperation, 2018A050506035).

\section{REFERENCES}

(1). Pushpavanam K; Narayanan E; Chang J; Sapareto S; Rege K A Colorimetric Plasmonic Nanosensor for Dosimetry of Therapeutic Levels of Ionizing Radiation. ACS Nano 2015, 9, 11540-11550. [PubMed: 26434692]

(2). Verma V; Simone CB; Mishra MV Quality of Life and Patient-Reported Outcomes Following Proton Radiation Therapy: A Systematic Review. J. Natl. Cancer Inst 2018, 110, 341-353.

(3). Pushpavanam K; Narayanan E; Rege K Molecular and Nanoscale Sensors for Detecting Ionizing Radiation in Radiotherapy. ChemNanoMat 2016, 2, 385-395.

(4). Jackman JA; Rahim Ferhan A; Cho N-J Nanoplasmonic Sensors for Biointerfacial Science. Chem. Soc. Rev 2017, 46, 3615-3660. [PubMed: 28383083]

(5). Wei T; Dong T; Wang Z; Bao J; Tu W; Dai Z Aggregation of Individual Sensing Units for Signal Accumulation: Conversion of Liquid-Phase Colorimetric Assay into Enhanced Surface-Tethered Electrochemical Analysis. J. Am. Chem. Soc 2015, 137, 8880-8883. [PubMed: 26149108]

(6). Goodman BA; Worasith N; Ninlaphruk S; Mungpayaban H; Deng W Radiation Dosimetry Using Alanine and Electron Paramagnetic Resonance (EPR) Spectroscopy: A New Look at an Old Topic. Appl. Magn. Reson 2017, 48, 155-173.

(7). Van Hoey O; Salavrakos A; Marques A; Nagao A; Willems R; Vanhavere F; Cauwels V; Nascimento LF Radiation Dosimetry Properties of Smartphone CMOS Sensors. Radiat. Prot. Dosim 2016, 168, 314-321.

(8). Rodríguez-Lorenzo L; de la Rica R; Álvarez-Puebla RA; Liz-Marzán LM; Stevens MM Plasmonic Nanosensors with Inverse Sensitivity by Means of Enzyme-Guided Crystal Growth. Nat. Mater 2012, 11, 604. [PubMed: 22635043]

(9). Brasiliense V; Patel AN; Martinez-Marrades A; Shi J; Chen Y; Combellas C; Tessier G; Kanoufi F Correlated Electrochemical and Optical Detection Reveals the Chemical Reactivity of Individual Silver Nanoparticles. J. Am. Chem. Soc 2016, 138, 3478-3483. [PubMed: 26900633]

(10). Inamdar S; Pushpavanam K; Lentz JM; Bues M; Anand A; Rege K Hydrogel Nanosensors for Colorimetric Detection and Dosimetry in Proton Beam Radiotherapy. ACS Appl. Mater. Interfaces 2018, 10, 3274-3281. [PubMed: 29303547]

(11). Yang X; Yang M; Pang B; Vara M; Xia Y Gold Nanomaterials at Work in Biomedicine. Chem. Rev 2015, 115, 10410-10488. [PubMed: 26293344]

(12). Edgar JA; McDonagh AM; Cortie MB Formation of Gold Nanorods by a Stochastic "Popcorn" Mechanism. ACS Nano 2012, 6, 1116-1125. [PubMed: 22301937]

(13). Lan X; Su Z; Zhou Y; Meyer T; Ke Y; Wang Q; Chiu W; Liu N; Zou S; Yan H; Liu Y Programmable Supra-Assembly of a DNA Surface Adapter for Tunable Chiral Directional SelfAssembly of Gold Nanorods. Angew. Chem., Int. Ed 2017, 56, 14632-14636.

(14). Gonzáez-Rubio G; Díaz-Núñez P; Rivera A; Prada A; Tardajos G; González-Izquierdo J; Bañares L; Llombart P; Macdowell LG; Alcolea Palafox M; Liz-Marzán LM; Peña-Rodríguez O; Guerrero-Martínez A Femtosecond Laser Reshaping Yields Gold Nanorods with Ultranarrow Surface Plasmon Resonances. Science 2017, 358, 640. [PubMed: 29097547] 
(15). Lippok N; Villiger M; Albanese A; Meijer EFJ; Chung K; Padera TP; Bhatia SN; Bouma BE Depolarization Signatures Map Gold Nanorods within Biological Tissue. Nat. Photonics 2017, 11, 583. [PubMed: 29201136]

(16). Ali MRK; Wu Y; Tang Y; Xiao H; Chen K; Han T; Fang N; Wu R; El-Sayed MA Targeting Cancer Cell Integrins Using Gold Nanorods in Photothermal Therapy Inhibits Migration through Affecting Cytoskeletal Proteins. Proc. Natl. Acad. Sci. U.S.A 2017, 114, E5655-E5663. [PubMed: 28652358]

(17). Lien M-B; Kim J-Y; Han M-G; Chang Y-C; Chang Y-C; Ferguson HJ; Zhu Y; Herzing AA; Schotland JC; Kotov NA; Norris TB Optical Asymmetry and Nonlinear Light Scattering from Colloidal Gold Nanorods. ACS Nano 2017, 11, 5925-5932. [PubMed: 28510416]

(18). Coronado-Puchau M; Saa L; Grzelczak M; Pavlov V; Liz-Marzán LM Enzymatic Modulation of Gold Nanorod Growth and Application to Nerve Gas Detection. Nano Today 2013, 8, 461-468.

(19). Guo X; Zhang Q; Sun Y; Zhao Q; Yang J Lateral Etching of Core-Shell Au@ Metal Nanorods to Metal-Tipped Au Nanorods with Improved Catalytic Activity. ACS Nano 2012, 6, 1165-1175. [PubMed: 22224460]

(20). Kulkarni AP; Noone KM; Munechika K; Guyer SR; Ginger DS Plasmon-Enhanced Charge Carrier Generation in Organic Photovoltaic Films Using Silver Nanoprisms. Nano Lett 2010, 10, 1501-1505. [PubMed: 20235514]

(21). Eckhardt S; Brunetto PS; Gagnon J; Priebe M; Giese B; Fromm KM Nanobio Silver: Its Interactions with Peptides and Bacteria, and its Uses in Medicine. Chem. Rev 2013, 113, 47084754. [PubMed: 23488929]

(22). Li Y; Li Z; Gao Y; Gong A; Zhang Y; Hosmane NS; Shen Z; Wu A “Red-to-Blue" Colorimetric Detection of Cysteine via Anti-Etching of Silver Nanoprisms. Nanoscale 2014, 6, 10631-10637. [PubMed: 25083798]

(23). Shahjamali MM; Bosman M; Cao S; Huang X; Saadat S; Martinsson E; Aili D; Tay YY; Liedberg B; Loo SCJ; Zhang H; Boey F; Xue C Gold Coating of Silver Nanoprisms. Adv. Funct. Mater 2012, 22, 849-854.

(24). Yang X; Yu Y; Gao Z A Highly Sensitive Plasmonic DNA Assay Based on Triangular Silver Nanoprism Etching. ACS Nano 2014, 4902-4907. [PubMed: 24766422]

(25). Tan K; Guang Y; Chen H; Shen P; Huang Y; Xia Y Facet Dependent Binding and Etching: UltraSensitive Colorimetric Visualization of Blood Uric Acid by Unmodified Silver Nanoprisms. Biosens. Bioelectron 2014, 59, 227-232. [PubMed: 24732599]

(26). Saa L; Grinyte R; Sánchez-Iglesias A; Liz-Marzán LM; Pavlov V Blocked Enzymatic Etching of Gold Nanorods: Application to Colorimetric Detection of Acetylcholinesterase Activity and Its Inhibitors. ACS Appl. Mater. Interfaces 2016, 8, 11139-11146. [PubMed: 27070402]

(27). Zhang Z; Chen Z; Pan D; Chen L Fenton-Like Reaction-Mediated Etching of Gold Nanorods for Visual Detection of Co2+. Langmuir 2015, 31, 643-650. [PubMed: 25486441]

(28). Liu X; Zhang S; Tan P; Zhou J; Huang Y; Nie Z; Yao S Plasmonic Blood Glucose Monitor Based on Enzymatic Etching of Gold Nanorods. Chem. Commun 2013, 49, 1856-1858.

(29). Chen Z; Zhang Z; Qu C; Pan D; Chen L Highly Sensitive Label-Free Colorimetric Sensing of Nitrite Based on Etching of Gold Nanorods. Analyst 2012, 137, 5197-5200. [PubMed: 22970427]

(30). Tao Y; Ju E; Liu Z; Dong K; Ren J; Qu X Engineered, Self-Assembled Near-Infrared Photothermal Agents for Combined Tumor Immunotherapy and Chemo-Photothermal Therapy. Biomaterials 2014, 35, 6646-6656. [PubMed: 24818880]

(31). Marta B; Jakab E; Potara M; Simon T; Imre-Lucaci F; Barbu-Tudoran L; Popescu O; Astilean S Pluronic-Coated Silver Nanoprisms: Synthesis, Characterization and Their Antibacterial Activity. Colloids Surf., A 2014, 441, 77-83.

(32). Busbee BD; Obare SO; Murphy CJ An Improved Synthesis of High-Aspect-Ratio Gold Nanorods. Adv. Mater 2003, 15, 414-416.

(33). Caswell KK; Wilson JN; Bunz UHF; Murphy CJ Preferential End-to-End Assembly of Gold Nanorods by Biotin-Streptavidin Connectors. J. Am. Chem. Soc 2003, 125, 13914-13915. [PubMed: 14611200] 
(34). David L; Bhandavat R; Barrera U; Singh G Silicon Oxycarbide Glass-Graphene Composite Paper Electrode for Long-Cycle Lithium-Ion Batteries. Nat. Commun 2016, 7, No. 10998.

(35). Wang J; Zhong H; Wang Z; Meng F; Zhang X Integrated Three-Dimensional Carbon Paper/ Carbon Tubes/Cobalt-Sulfide Sheets as an Efficient Electrode for Overall Water Splitting. ACS Nano 2016, 10, 2342-2348. [PubMed: 26783885]

(36). Park H; Yu S; Yang K; Jin Y; Cho A-N; Kim J; Lee B; Yang HS; Im SG; Cho S-W Paper-Based Bioactive Scaffolds for Stem Cell-Mediated Bone Tissue Engineering. Biomaterials 2014, 35, 9811-9823. [PubMed: 25241158]

(37). Chaiyo S; Siangproh W; Apilux A; Chailapakul O Highly Selective and Sensitive Paper-Based Colorimetric Sensor Using Thiosulfate Catalytic Etching of Silver Nanoplates for Trace Determination of Copper Ions. Anal. Chim. Acta 2015, 866, 75-83. [PubMed: 25732695]

(38). Noor MO; Shahmuradyan A; Krull UJ Paper-Based Solid-Phase Nucleic Acid Hybridization Assay Using Immobilized Quantum Dots as Donors in Fluorescence Resonance Energy Transfer. Anal. Chem 2013, 85, 1860-1867. [PubMed: 23272728]

(39). Ornatska M; Sharpe E; Andreescu D; Andreescu S Paper Bioassay Based on Ceria Nanoparticles as Colorimetric Probes. Anal. Chem 2011, 83, 4273-4280. [PubMed: 21524141]

(40). Kacprowska A; Jassem J Hypofractionated Radiotherapy for Early Breast Cancer: Review of Phase III Studies. Rep. Pract. Oncol. Radiother 2012, 17, 66-70. [PubMed: 24377002]

(41). Matsuoka S; Ballif BA; Smogorzewska A; McDonald ER; Hurov KE; Luo J; Bakalarski CE; Zhao Z; Solimini N; Lerenthal Y; Shiloh Y; Gygi SP; Elledge SJ ATM and ATR Substrate Analysis Reveals Extensive Protein Networks Responsive to DNA Damage. Science 2007, 316, 1160. [PubMed: 17525332]

(42). Ziv Y; Bielopolski D; Galanty Y; Lukas C; Taya Y; Schultz DC; Lukas J; Bekker-Jensen S; Bartek J; Shiloh Y Chromatin Relaxation in Response to DNA Double-Strand Breaks Is Modulated by a Novel ATM- and KAP-1 Dependent Pathway. Nat. Cell Biol 2006, 8, 870. [PubMed: 16862143]

(43). Iyengar S; Farnham PJ KAP1 Protein: An Enigmatic Master Regulator of the Genome. J. Biol. Chem 2011, 286, 26267-26276. [PubMed: 21652716] 


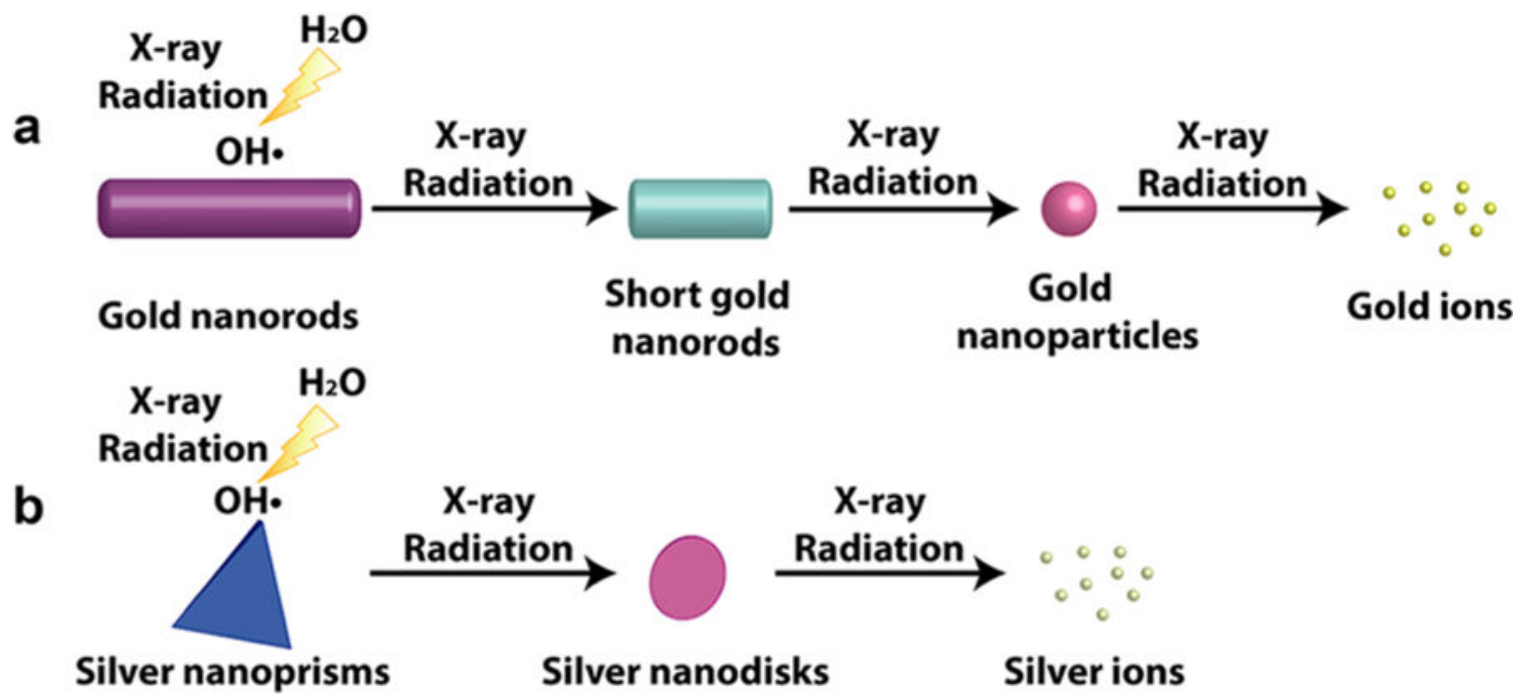

Figure 1.

Schematic illustration of radiation-based etching of gold nanorods and silver nanoprisms. (a) Radiation-mediated etching of gold nanorods leads to the formation of short gold nanorods and finally to the gold nanoparticles and gold ions. (b) Radiation-mediated etching of silver nanoprisms leads to the formation of smaller round Ag nanodisks and finally to the silver ions. 

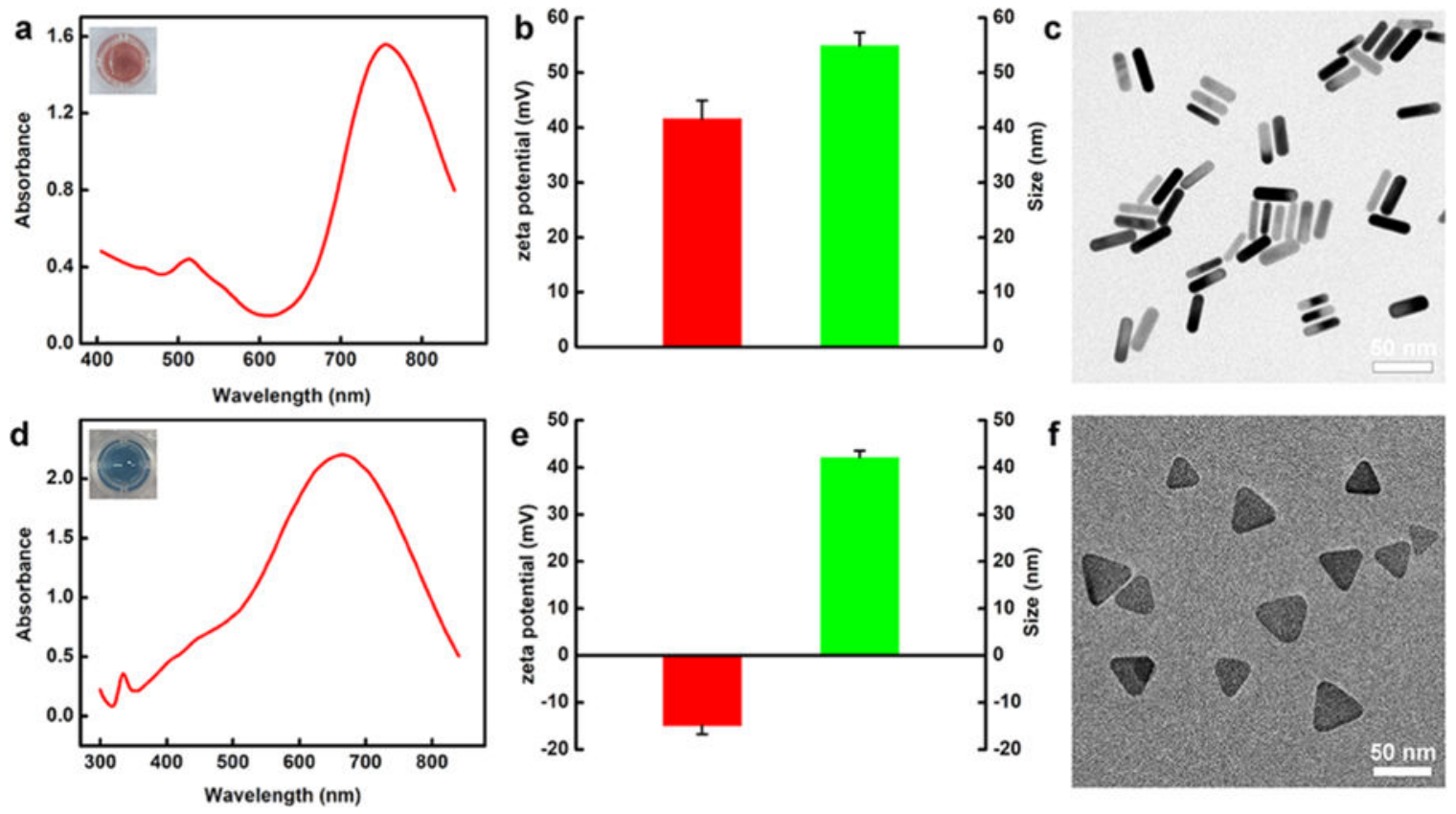

Figure 2.

Characterization of AuNRs and AgNPRs. Characterization of $(\mathrm{a}-\mathrm{c})$ gold nanorods and $(\mathrm{d}-\mathrm{f})$ silver nanoprisms, including absorbance spectra $(a, d), \zeta$-potentials and hydrodynamic sizes $(b, e)$, and transmission electron microscopy (TEM) images (c, f) of the nanoparticles. In (b) and (e), the red columns represent the $\zeta$-potential and the green columns represent the hydrodynamic size. 

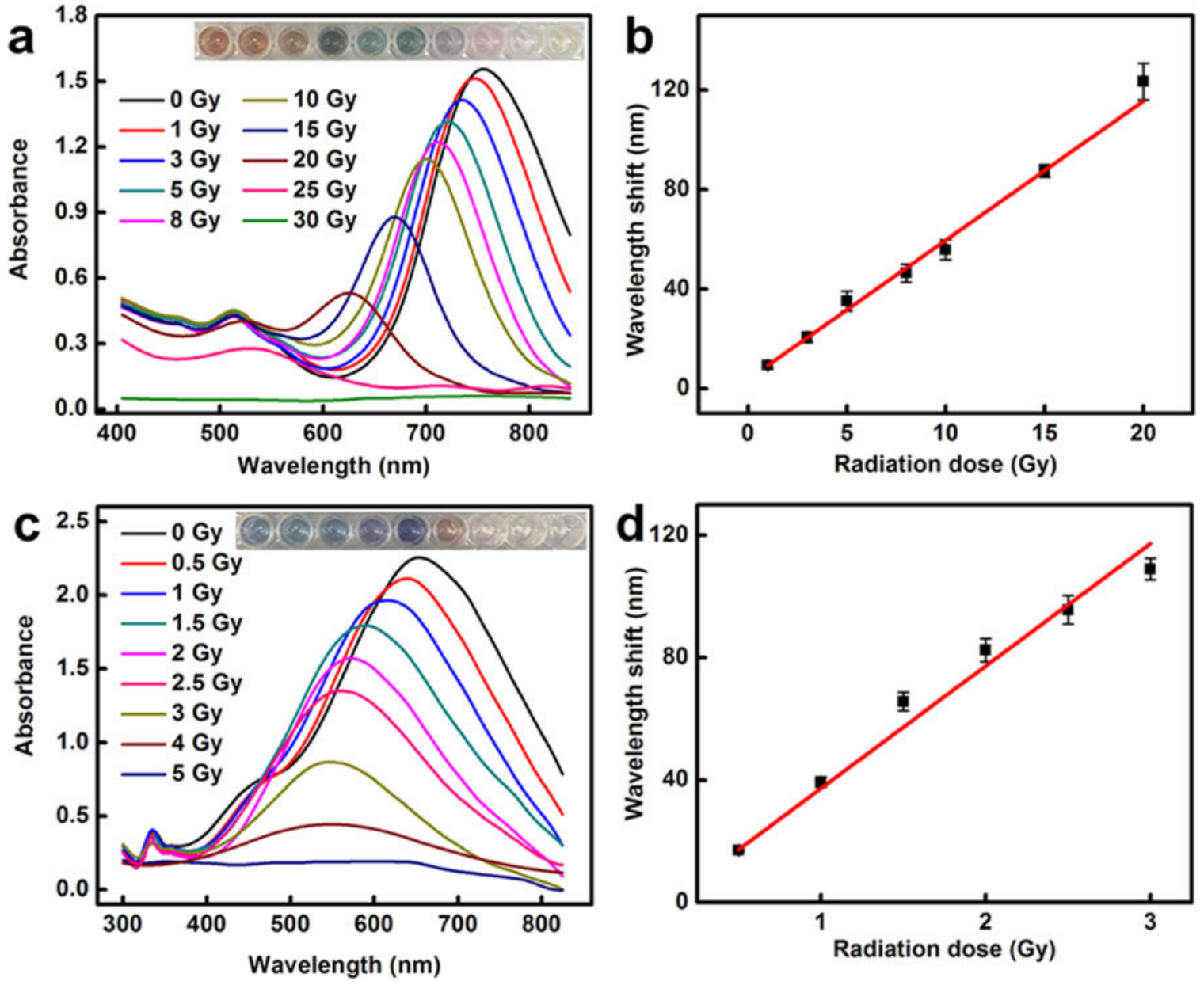

Figure 3.

Absorption spectra. Absorption spectra and linear calibration curves of ( $a, b)$ gold nanorods and (c, d) silver nanoprisms irradiated with a range of X-ray doses (Gy). Absorption spectra of gold nanorods irradiated with increasing X-ray doses $(0,1,3,5,8,10,15,20,25$, and 30 Gy) (a). The linear plot of longitudinal peak shift as a function of radiation dose from 1 to 20 Gy (b). Absorption spectra responses of the silver nanoprisms irradiated with different X-ray doses $(0,0.5,1,1.5,2,2.5,3,4$, and 5 Gy) (c). Linear plot of wavelength shift as a function of the radiation dose from 0.5 to $3 \mathrm{~Gy}(\mathrm{~d})$. 

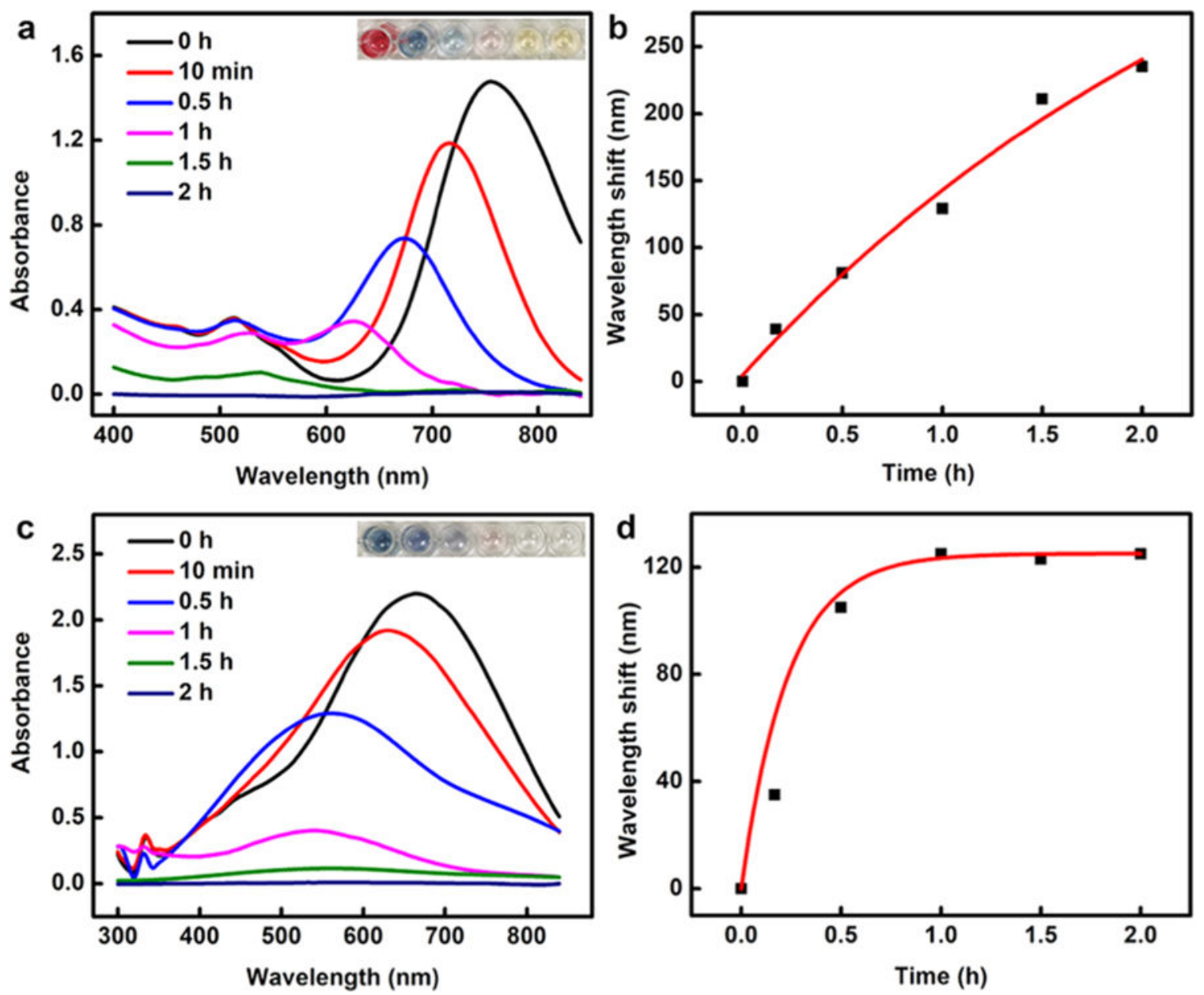

Figure 4.

(a) Absorption spectra of gold nanorods irradiated with X-ray (30 Gy) at different times. (b) Time-dependent longitudinal peak shift of gold nanorods as a function of $\mathrm{X}$-ray radiation (30 Gy). (c) Absorption spectra of silver nanoprisms irradiated with X-ray (5 Gy) at different times. (d) Time-dependent wavelength shift of silver nanoprisms as a function of $\mathrm{X}$-ray radiation (5 Gy). 


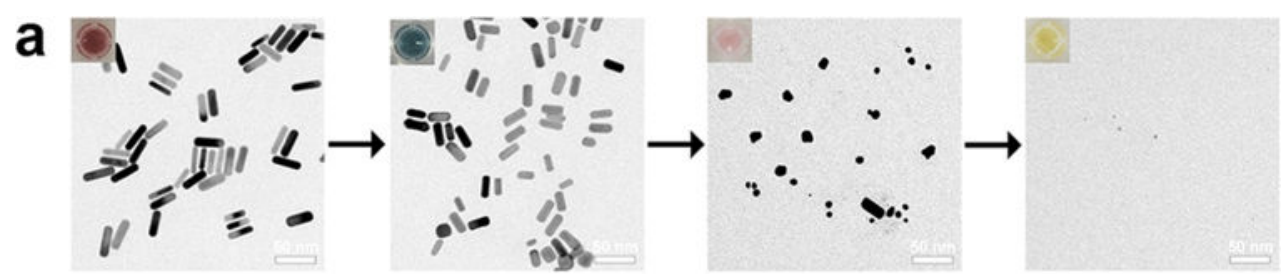

b

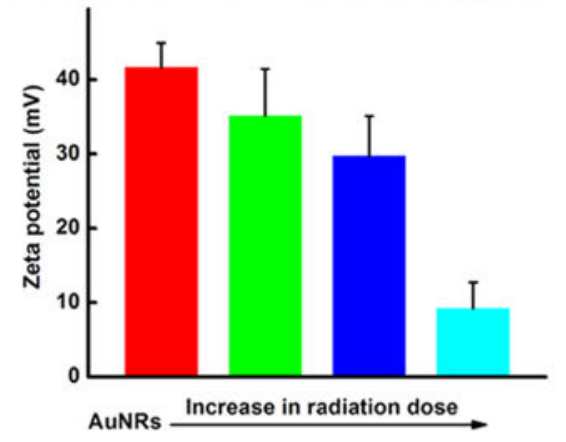

C

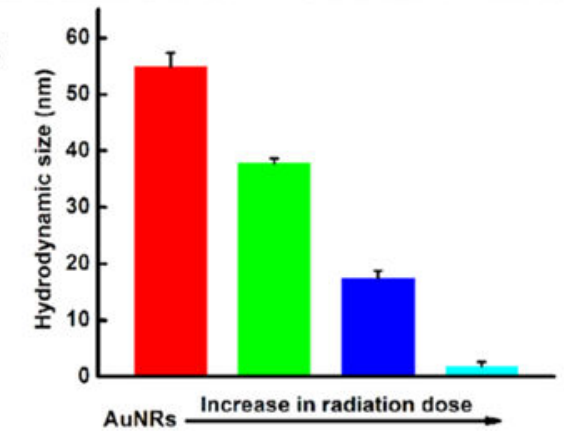

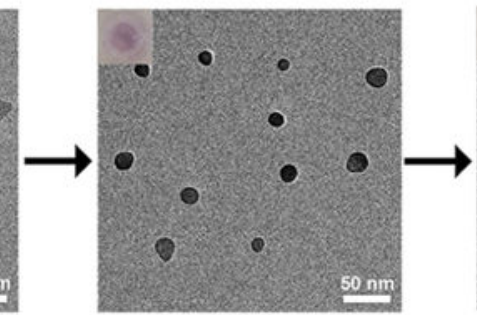

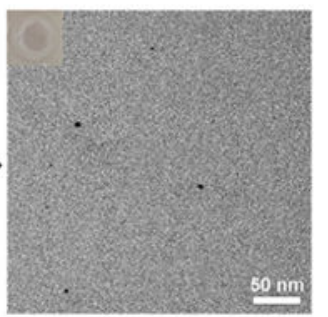

e
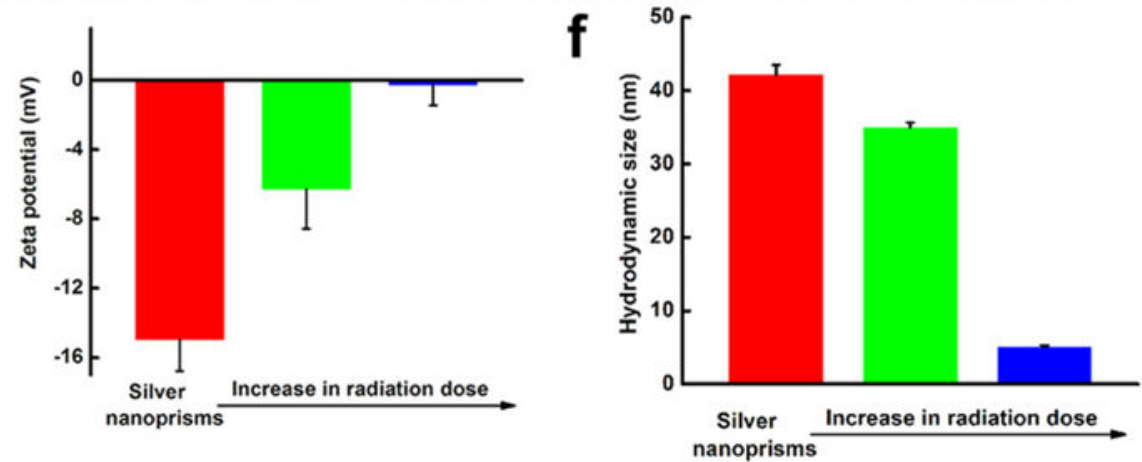

Figure 5.

Shapes and properties of nanoparticles upon irradiation. TEM images (a, d), $\zeta$-potentials (b, e), and hydrodynamic sizes $(c, f)$ of gold nanorods $(a-c)$ and silver nanoprisms $(d-f)$ after different radiation doses (gold nanorods: $0 \mathrm{~Gy} \rightarrow 5 \mathrm{~Gy} \rightarrow 20 \mathrm{~Gy} \rightarrow 30 \mathrm{~Gy}$; silver nanoprisms: 0 Gy $\rightarrow 2.5$ Gy $\rightarrow 5$ Gy). 

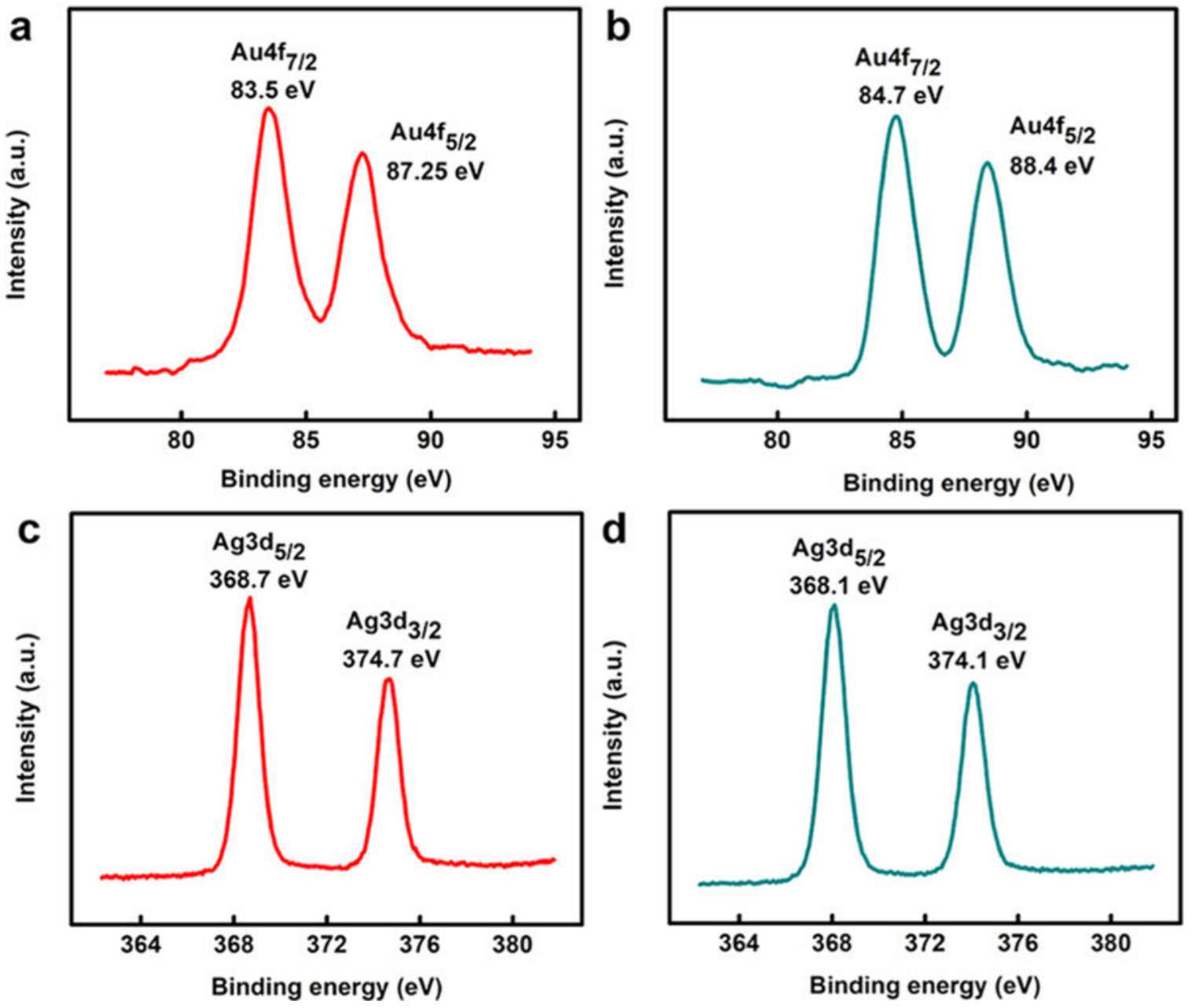

Figure 6.

(a, b) XPS spectra of gold nanorods (a) before and (b) after irradiated with X-ray (30 Gy).

(c, d) XPS spectra of silver nanoprisms (c) before and (d) after X-ray irradiation (5 Gy). 


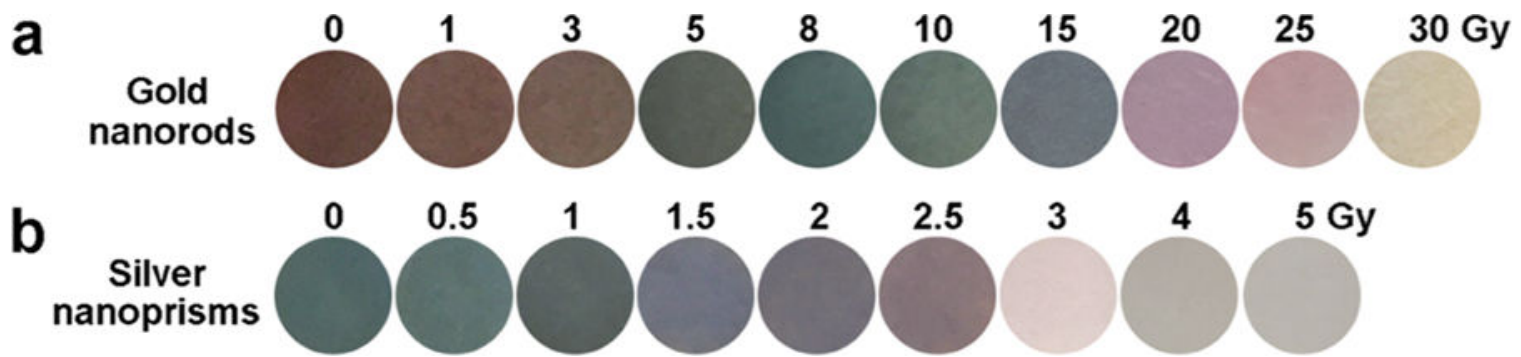

Figure 7.

Paper-based colorimetric assay. (a) Gold nanorods and (b) silver nanoprisms were irradiated at the indicated doses and spotted on the filter paper (gold nanorods: $0,1,3,5,8,10,15,20$, 25 , and $30 \mathrm{~Gy}$, silver nanoprisms: $0,0.5,1,1.5,2,2.5,3,4$, and $5 \mathrm{~Gy}$ ). 


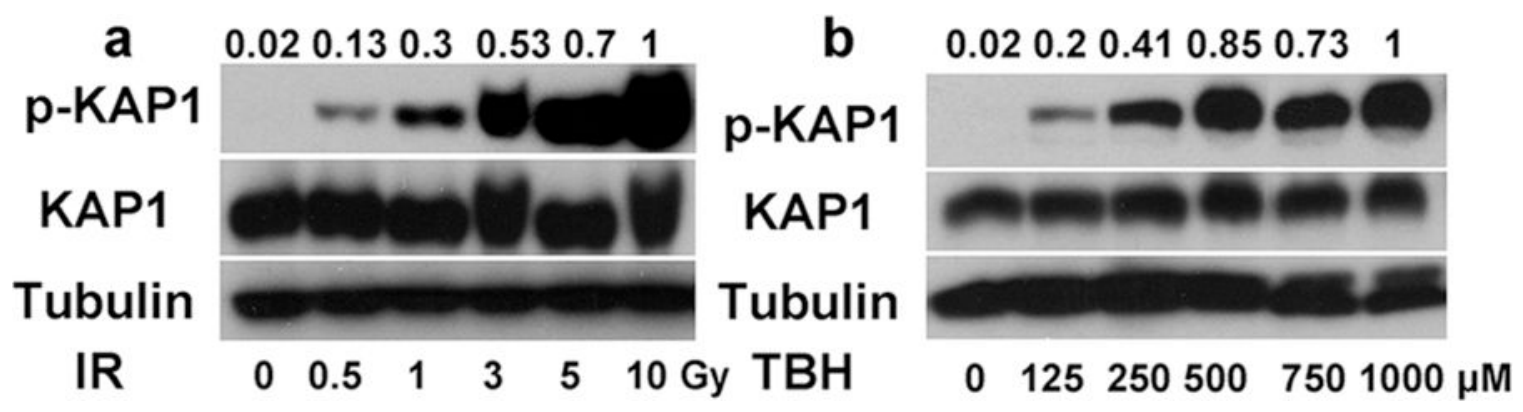

Figure 8.

ATM activation by IR and ROS. Mouse embryonic fibroblasts (MEFs) were treated with (a) IR (0-10 Gy) or (b) TBH (0-1000 $\mu \mathrm{M})$. Cellular protein extracts were prepared for $2 \mathrm{~h}$ by following radiation exposure or $2 \mathrm{~h}$ after $\mathrm{TBH}$ treatment, then immunoblotted with the indicated antibodies: phospho-KAP1 (pKAP1), KAP1, and tubulin. The increase in the intensity of pKAP1 is indicated on top of the Western blots. 